\title{
Virulence Factor and Pathogenicity of Candida albicans in Oral Candidiasis
}

\author{
Abdillah Imron Nasution
}

\begin{abstract}
Candida albicans is an opportunistic fungus causing various forms of candidiasis. However, under certain circumstances it is capable of becoming pathogenic. Pathogenicity of oral candidiasis is a complex process and there is no one factor that can be regarded as the direct cause. This review aims to explain the virulence factors of Candida albicans in oral candidiasis infection and its relation to homeostasis in the mouth. Virulence factors of Candida albicans which is closely related to the nature of pathogens include: adherence and coaggregation, interference of immune system, phenotype switching and several supporting factors such as antibiotic resistance and immunomodulating.
\end{abstract}

Keywords: Candida albicans, Oral candidiasis, Virulence factor, Pathogenicity.

How to cite this article: Nasution Al. Virulence Factor and Pathogenicity of Candida albicans in Oral Candidiasis. World $\mathrm{J}$ Dent 2013;4(4):267-271.

Source of support: Nil

Conflict of interest: None declared

\section{INTRODUCTION}

Candida albicans is an opportunistic fungus causing various forms of candidiasis. Genus of Candida albicans consists of number of species that associated with human disease. Candida albicans is a commensal living in various parts of the human body and animals, ${ }^{1}$ is the most common species found to cause oral candidiasis. Oral carrier rate in the normal population varies about $20 \%$. Candida albicans is a major organism associated with infection, but other species such as Candida parapsilosis and Candida krusei can also be pathogenic to humans. ${ }^{2}$

In humans, the Candida albicans is frequently isolated from mucosal surfaces in the mouth, esophagus and gastrointestinal tract, therefore Candida albicans is often found as isolates from the oral cavity, as normal flora and cause candidiasis with various forms of manifestations and clinical symptoms. ${ }^{1}$

Pathogenicity of oral candidiasis is a complex process and there is no one factor that is able to be the direct cause. ${ }^{3}$ Presence of supporting factors such as host or fungus is needed in the process of invasion of Candida albicans. Candida albicans infection is closely associated with virulence factors. Such diversity is an indication that in the mouth there are a variety of virulence factors of Candida albicans in oral candidiasis. ${ }^{4}$ Based on this problem, understanding the mechanisms of infection of Candida albicans will support, improve laboratory diagnosis and treatment of Candida albicans. The aim of this review is to describe virulence factors of Candida albicans in oral candidiasis infection and its relation to homeostasis of oral ecosystem.

\section{DISCUSSION}

\section{The Classification of Fungi}

Fungi are classified on the basis of the form of sexual reproduction, but at the sexual stage, fungi are difficult to be induced and rarely observed in clinical material. Most fungi produced through the formation of conidia in mitosis stage providing has same chromosome number. Conidia are produced through propagative form of asexual reproduction. Some of the fungi formed macroconidia and microconidia. There are three conidial structures, namely: arthroconidia, blastoconidia and chlamydoconidia. ${ }^{5}$

Arthroconidia or artrospora known as the structure resulting from fragmentation of a hyphae into individual cells, such as Coccidioides immitis. The second form, blastoconidia (chlamydospora) is a simple structure that is formed from the bud sprouting with subsequent separation of stem cells, such as found in yeast. The third form of conidia, chlamydoconidia or often referred to as chlamydospora shows the structure of the tip cells in enlarge hyphae and cause cell wall thickening. This structure is resistant to unfavorable environmental conditions and will germinate when conditions become more favorable for vegetative growth, such as Candida albicans. ${ }^{5}$

\section{Mycotic Infections}

It has been known that there are thousands of species of yeast and fungi, but only a 100 species cause disease in humans and animals (the others cause disease in plants). While only a species of dermatophytes and Candida albicans is often transmitted from one person to others. ${ }^{6}$ Human mycotic infections are grouped into superficial fungal infections, cutaneous, subcutaneous and profunda (or systemic). Superficial fungal infections, cutaneous or subcutaneous on the skin, hair and nails can become chronic and resistant to treatment but rarely affects the general health of the patient. The mycoses profunda caused by pathogenic fungi or opportunistic fungi that infected patients by immunologic disorders. The mycoses profunda can cause systemic disorder which can sometimes have fatal consequences. ${ }^{5}$ 


\section{Morphology of Candida albicans}

Candida albicans yeast appear as oval, buds, Gram-positive, size 2-3 $\times 4-6 \mu \mathrm{m}$ and has budded cells that resemble elongated hyphae (pseudohyphae). ${ }^{5}$ Candida albicans ferment glucose and maltose, produce acid and gas; ferment acid from sucrose, and do not react with lactose. The carbohydrate fermentation along with the properties and morphology distinguish colonies of other candida. ${ }^{6}$ The clinical appearance of Candida albicans especially in infants occurs in the lining mucosa of cheek and appear as white patches that are mostly composed of epithelial pseudomycelium, and there is only minimal erosion in lining area. Growth of Candida albicans in the mouth will be more fertile when accompanied by corticosteroids, antibiotics, high glucose levels and the immunodeficiency condition. ${ }^{7}$

\section{The Pathogenicity of Candida albicans}

Oral candidiasis is one of the manifestations of mucocutaneous infections in the oral mucosa that is triggered by physiological changes and disturbances of homeostasis in the immune system of patients. ${ }^{8}$ In other words, the integrity changes of host immune system is an important factor to determine, whether the Candida albicans will still behave as commensal on the mouth ecosystem or able to proliferate, resulting in oral candidiasis. This chronic condition, is caused by a defect of cellular immune response of patients. ${ }^{4}$

Potential of Candida albicans to form colonies, penetrate and cause infection in the mouth depends on the imbalance of virulent factors that determines the presence of microorganisms and certain defects of the host immune system. ${ }^{8}$ Manifestations of oral candidiasis, basically due to the opportunities that allow the Candida albicans can selectively express virulent factors that are required to exist for Candida albicans as pathogen. ${ }^{9}$ One pathogenic properties of Candida albicans is its ability to form chlamydospora. Chlamydospora is a refractile cell, thin-walled, and formed on the terminal or lateral of hyphae and occasionally on pseudohypha. ${ }^{1}$ The formation of chlamydospora is developed by the environment that has limited oxygen in circumstances of low temperatures. ${ }^{10}$ That means, the success of Candida albicans to exist as a pathogen in the mouth is closely associated with the expression of certain genes, which are required to adapt to the various phases of infection in the mouth.

\section{Virulence Factor of Candida albicans}

There are several virulence factors of Candida albicans in oral candidiasis infection and its relation to homeostasis of host in the mouth, namely:

\section{Adherence and Coaggregation}

\section{Adherence}

Virulence factor of Candida albicans are determined by cell wall. Cell wall plays an important role because this part directly interacts with host cells. Cell wall of Candida albicans contains substances that are essential for virulence, such as derivatives mannoprotein that have immunosuppressive properties to enhance the defense of candida against immune system of host. ${ }^{9,11}$ The mechanisms of Candida albicans infection are very complex, including adhesion and invasion, changes of cell shape morphology from yeast form into filaments form (hyphae), biofilm formation, and able to avoid from host immune cells. The ability of Candida albicans attached to host cells is an important factor in the early stages of colonization and infection. $^{10}$

Adhesion may occur due to the expression of various antigens on the cell surface that act as adhesion molecules. This expression was recognize as extracellular protein that found on the surface epithelial of cells host. ${ }^{12}$ Proteins that acts as a mediator of adhesion were classified as proteins serum (serum albumin and transferrin, fibrinogen, C3D complement fragments and $\mathrm{iC} 3 \mathrm{~b}$ complement fragment); extracellular matrix proteins (laminin, fibronectin, entactin, vitronectin, collagen); mannan adhesins and other binding proteins (mannan adhesins, hydrophobic proteins, fimbriae, plastic-binding protein, epithelial binding lectin-like protein, agglutinin-like proteins, adhesion on Streptococcus species, other bacteria), as well as adhesion to saliva. ${ }^{9}$

This adhesion will cause the transformation of Candida albicans from blastopore into chlamydospora form. The ability of Candida albicans transformed into pseudohyphae was one of important virulence factor. ${ }^{1}$ Hyphae have a higher virulence than the spore form because: first, it was bigger and more difficult to phagocyte by macrophage, consequently immune cell need other mechanisms to eliminate hyphae on infected tissue. ${ }^{12}$ Second, the presence of multiple blastoconidia points on the filament increasing a large amount of infectious elements. ${ }^{9}$ Adhesions may occur in epithelial cells of the mouth and polymethylmethacrylate (PMMA) denture materials with lectin-like component that acts as a mediator of adhesion. Mediator components will interact with mono- or disaccharide host. ${ }^{9}$ In hyphae, adhesion will more increasingly as big as lectin-like component to be taken out. ${ }^{13}$ Epithelial penetration by hyphae were facilitated by aspartyl proteinases and phospholipase. ${ }^{14}$

The penetration that occurs can disrupt the epithelial surface by extracellular neutralization and acid protease of Candida albicans opening an opportunity of Candida 
albicans to adhere. Candida albicans adhere to the mucosal surface, then compete other microorganisms saprophyte commensal in mouth for nutrients. If the place of microflora was disturbed, Candida albicans will grow up unstoppable to facilitated infectious. ${ }^{1,2}$

On candidiasis erythematous case, Candida albicans showed strong adherence to PMMA. ${ }^{15,16}$ According to several studies Candida adhesion onto PMMA-based resins is a common source of oral cavity infection and stomatitis. Denture installations were known as favorable environment making Candida albicans settle in the oral mucosa. ${ }^{17}$ Cells of tip or hyphae enlarged and cell walls was thickened. In other words, environmental conditions are one of important key role for Candida albicans conducted different morphological forms and morphogenic change to increasing of pathogenicity.

\section{Coaggregation}

Coaggregation usually occurs in chronic atrophic candidiasis and effect of mediation $S$. mutans that found in denture surface. Streptococcus mutans plays an important role in the colonization of Candida albicans. Previous study stated that $C$. albicans and $S$. mutans enhance binding to each other and to surfaces in the presence of sucrose. Scanning electron microscopy images also reveal glucan-like structures that form between cocci and yeasts, suggesting that glucans may play a role in mediating their interactions. ${ }^{18}$

Lipoprotein known as mediator of adhesion of S. sanguis that has a dual function in recognizing by receptors of carbohydrate and some receptors that presented in saliva. Carbohydrate receptors on adhesion can be purified by five types of streptococci in the oral cavity. This shared aggregation can specifically inhibit coaggregation of another type. $^{2}$

Other research states that Candida albicans ATCC 10261 and CA2 bind to oral bacteria such as Streptococci. ${ }^{19}$ Cell persistence of Candida albicans in the oral cavity may be possible because these fungi expressed different antigens on cell surface. These antigens act as adhesion molecules that recognize extracellular proteins same as found on the surface of epithelial cells. This interaction causing the cell Candida albicans adhere to the mucosal surface and allow the invasion of the epithelial tissue. This interaction leads to the transformation of yeast cells to form pseudohyphae blastopore.

\section{Interference of Immune System}

\section{Interference of $\operatorname{s} \lg A$}

Interference of $\operatorname{sIgA}$ begins with epithelial penetration by hyphae and supported by aspartyl proteinase and phospholipase to reducing the production of saliva. ${ }^{14}$ Moreover, the effect of pathogenicity of Candida albicans and coaggregates with $S$. mutants improving of acid production which will be followed by decreased SIgA and finally disadvantage for immunology responses. ${ }^{8,12}$

\section{Interference of Complement}

The complement system is a mediator to opsonization and chemotaxis by preparing antigen for phagocytosis. The only certain of adhesion molecules expressed on the cell surface of pseudohyphae. Adhesion molecule by complement interference is a receptor of $\mathrm{C} 3 \mathrm{D}$ and $\mathrm{iC} 3 \mathrm{~b}$. Expression of protein molecules on the cell surface of Candida albicans will affect the pathogenicity of Candida albicans. ${ }^{2}$

\section{Interference of Phagocytosis}

Dimorphic trait virulence factor of Candida albicans was instrumental in phagocytosis interference, even some researchers claiming it as pleomorfic. ${ }^{8}$ Dynamic morphological properties is a way to adapt Candida albicans to surroundings environment. The two main forms of Candida albicans is a yeast form and pseudohyphae form which are also referred to as mycelium. This change is known as process of adaption of Candida albicans to environmental from commensal into pathogen. ${ }^{7}$ In circumstances pathogen, Candida albicans are more commonly found in the form of mycelium or pseudohyphae or filaments than spores ones. Growth and changes in the form of yeast into a more invasive hyphae also affected the cellular immunity.

The occurrence of phagocytosis interference will result in decreasing the serum levels of complement and inability of PMN. Phagocytosis by PMN and macrophages can be inhibited by peptides and acid production of extracellular glycoprotein of Candida albicans. Systemic candidiasis is also associated with the absence of myeloperoxidase and hydrogen peroxide in phagocytic cells. Therefore, the function of phagocytic cells cannot process the macrophage activating factor (MAF). With the absence of MAF, macrophages cannot adhere to cell Candida albicans through mannose receptor membrane or by attaching vitronectin (a cell adhesion glycoprotein). Macrophages eventually will not be able to produce nitrogen monoxide that is known as fungicidal. ${ }^{11}$

\section{Phenotype Switching}

Aspects of molecular phenotype has not clearly revealed. Some researcher supposed that it is more common in the virulent strain compared to flora normal. ${ }^{3}$ Example, candidiasis in patients with AIDS. ${ }^{9}$ Changes in the phenotype of a filament will penetrate into epithelium and role in 
infection and spread of Candida albicans to cells of host. Candida albicans that can form biofilms is thought to be involved in the attacking of host cells and is associated with resistance to antifungal properties. Morphology of Candida abicans supports the composition changes. Colonies of some strains of Candida albicans is able to change the shape. ${ }^{20}$ Adaptation will be conducted in accordance with the location in the oral cavity as a commensal or a pathogen. This will affect the fat content and sterols in the cell wall affected the attachment of Candida albicans to mucosal epithelium, consequently improving antigen expression and susceptibility to antifungal preparations. ${ }^{3}$

\section{Supporting Factor}

\section{Antibiotic Resistance}

Candida albicans not only attached into mucosa, but is also able to penetrate. Aspartyl proteinase enzymes will support Candida albicans having early stages to penetrate the layers of keratinized-mucocutaneous tissue. ${ }^{14}$ When the complement of $\mathrm{C} 3$ component receptors blocked by fungal cell interaction, host will be influenced by external factors, such as the use of antibiotics. Antibiotics will suppress the growth of competitive microorganisms for Candida albicans. These conditions make Candida albicans more easier to form colonies.

\section{Others}

There are two main aspects of the interaction between host and agent namely, host immunomodulating immune response and adhesion of agent into host cell. Immunomodulatory have potential ability to candida cells to modulate immunological system of host. This condition is known as trigger for host so as to whether increase or decrease the host immune response. ${ }^{21}$ Substances of cell wall such as chitin, glucans and mannoprotein were involved in immunomodulation response to producing a number of proteins known as heat shock proteins (HSP). ${ }^{9}$ There are two families of HSP known, namely HSP90 and HSP70. ${ }^{9}$ These conditions were advantage for Candida albicans. HSPs also play a key role in the growth process of Candida albicans to stimulate host immune response. Several protease enzymes known are only secreted by Candida species at the systemic infection as penetration of hyphae to epithelial cells known are supported by aspartyl proteinases. ${ }^{14}$

\section{CONCLUSION}

1. Virulence factors of Candida albicans which is closely related to the nature of pathogens include: adherence and coaggregation, interference of immune system, phenotype switching, and several supporting factors such as antibiotic resistance and immunomodulation.

2. Immune components that are involved in the virulence and pathogenicity of Candida albicans are sIgA, complement, phagocytes and macrophages.

3. Adaptation will affect the fat content and sterols in the cell wall which will give effect to the attachment to the mucosal epithelium, antigen expression and susceptibility to antifungal preparations.

4. Changes in the phenotype of a filament form allows Candida albicans to penetrate into epithelium and has role in infection and spread in host cell.

5. Antibiotics suppress the growth of microorganisms that are competitive against Candida albicans, making candida easier to form colonies.

6. HSP (Hsp90 and Hsp70) also play a role in stimulating a response in the growth process.

\section{REFERENCES}

1. Matthews RC. Pathogenicity determinant of Candida albicans: potential targets for immunotherapy? Microbiology 1994;140:1505-1511.

2. Fonzi WA, Calderon RA. Virulence factor of Candida albicans. Trends Microbiol 2001:327:333-339.

3. Gillmore BJ, Retsinas EM, Lorenz JS. An C3b receptor of Candida albicans structure, function, and correlates for pathogenicity. $\mathrm{J}$ Infect Dis 1998;157:38-34.

4. Bachtiar BM. Some factors affecting virulence pathogenicity of Candida albicans candidiasis in mouth. J Dent Univ Indonesia 1997;4:704-706.

5. Brooks GF, Carroll KC, Butel JS, Morse SA. Jawetz, Melnick, Adelberg's medical microbiology. 24th ed. McGraw-Hill; 1995. p. 608-610.

6. Diamod RD. Interaction of phagocityc cell with candida and other opportunistic fungi. Arch Med Res 1993;24:361-369.

7. Ashman RB, Papadimitriou JM. Murine candidiasis: strain depedence of host response after immunization. Immunol Cell Biol 1998;66:231-237.

8. Greenfield RA. Host defence system interaction with Candida albicans. J Med Vet Mycol 1992;30:89-104.

9. Chaffin WL, Lopez-Ribot JL, Casanova M, Gozalbo D, Martínez JP. Cell wall and secreted proteins of candida albicans: identification, function and expression. Microbiol Mol Biol Rev 1998;62:130-180.

10. Bryan MG, Libow L. Candidiasis (chronic mucocutaneous). E Med J 2001 Jun;2(6). Available from: http://www.emedicine.com.

11. Vazquez-Torres A, Balish E. Macrophage in resistance to candidiasis. Microbiol Mol Biol Rev 1997;61:170-192.

12. O'Sullivan JM, Jenkinson HF, Cannon RD. Adhesion of Candida albicans to oral streptococci is promoted by selective adsorption of salivary proteins to the streptococcal cell surface. Microbiology 2000;146:41-48.

13. Ebisu S, Nakae H, Okada H. Coaggregation of Eikenella corrodens with oral bacteria mediated by bacterial lectin-like substance. Adv Dent Res 1988 Nov;2(2):323-327. 
14. Ashman RB, Papadimitriou JM. Production and function of cytokines in natural and acquired immunity to Candida albicans infection. Microbiol Rev 1995;59:646-672.

15. Vasilas A, Molina L, Hoffman M, Haidaris CG. The influence of morphological variation on Candida albicans adhesion to denture acrylic in vitro. Arch Oral Biol 1992;37:613-622.

16. Edgerton M, Scannapieco FA, Reddy MS, Levine MJ. Human submandibular-sublingual saliva promotes adhesion of Candida albicans to polymethylmethacrylate. Infect Immun 1993;61:26442652.

17. Yamauchic M, Yamamoto K, Waka-bayashic M, Kawano J. In vitro adhérence of microorganism to denture base resins with different surface texture. Dent Mater J 1990;9(1):19-24.

18. Branting C, Sund ML, Linder LE. The influence of Streptococcus mutans on adhesion of Candida albicans to acrylic surfaces in vitro. Arch Oral Biol 1989;34:347-353.
19. Holmes AR, Gopal PK, Jenkinson HF. Adherence of Candida albicans to a cell surface polysaccharide receptor on Streptococcus gordonii. Infect Immun 1995 May;63(5):1827-1834.

20. Soll DR. High frequency switching in Candida albicans. Clin Microbiol Rev 1992;5:183-203.

21. Cassone A. Immunogenic and immunomodulatory properties of mannoproteins from Candida albicans. Can J Bot 1995;73(S1):1192-1198.

\section{ABOUT THE AUTHOR}

\section{Abdillah Imron Nasution}

Department of Oral Biology, Faculty of Medicine, Study of Dentistry Program, University of Syiah Kuala, Aceh, Indonesia Phone: 08126988519, e-mail: abdillah@karstaceh.com 